\title{
ESTUDO DO ATENDIMENTO DE ENFERMAGEM INSTITUCIONAL A CRIANÇAS DE 0 A 5 ANOS
}

Nelice Ferreira Moura*

MOURA, N.F. Estudo do atendimento de enfermagem institucional a crianças de 0 a 5 anos. Rev. Saúde vúbl.. S. Paulo, 15:371-8, 1981.

RESUMO: Foi realizada avaliação operacional sobre a implantação do atendimento de enfermagem institucional à criança de 0 a 5 anos realizado de outubro de 1977 a outubro de 1978 no Centro de Saúde Experimental da Escola Paulista de Medicina, São Paulo (Brasil). Focaliza-se não só o problema gerado desde a implantação desta atividade (a maioria dos atendimentos de enfermagem são encaminhados para consulta médica), mas também procura conhecer a opiniảo da clientela sobre esta nova modalidade de assistência de saúde à comunidade.

UNITERMos: Centros de Saúde. Crianças, assistência. Enfermagem, assistência. Avaliação.

\section{INTRODUÇAO}

O Programa de Assistência à Criança da Secretaria da Saúde do Estado de Săo Paulo foi implantado em 1977, com a finalidade de, entre outras atividades, prever 0 atendimento de enfermagem institucional **.

Este atendimento deveria ser realizado por pessoal auxiliar, à crianças sadias com a finalidade de se acompanhar o crescimento e desenvolvimento da criança.

Conforme preconizava a Secretaria da Saúde do Estado de São Paulo, foi implantada a programação no Centro de Saúde da Escola Paulista de Medicina (CSEPM), em cutubro de 1977. Nessa ocasião, o Centro de Saúde encontrava-se em déficit de recursos humanos, não sendo possivel realizar um programa de treinamento adequado.
Visto que esse tipo de atendimento caracterizava-se como de suma importância, no contexto de assistência de saúde simplificada no campo da saúde pública, procurou-se levantar dados que servissem de subsidios para a adequada sistematização da atividade.

A disciplina de Enfermagem de Saúde Pública da Faculdade de Saúde Pública da USP 2 sugere dentro da assistência de enfermagem:

- prestar especial atenção de enfermagem materno-infantil, oferecendo consulta de enfermagem a gestantes e crianças sadias, de acordo com padróes e normas estabelecidos.

- Oferecer atendimento de enfermagem institucional e domiciliário prestado

\footnotetext{
* Da Unidade de Enfermagem do Centro de Saúde Experimental da Escola Paulista de Me.icina (CSEPM) - Rua Pedro de Toledo, 840 - 04039 - São Paulo, SP - Brasil.

* Conjunto de ações prestadas por pessoal auxiliar a pessoas sadias de forma sistemática e completa, especificamente relacionadas com o controle do crescimento e desenvolvimento da criança sadia que não requer atenção imediata do médico ou da enfermeira.
} 
MOURA, N. F. Estudo do atendimento de enfermagem institucional a crianças de 0 a 5 anos. Rev. Saude públ., S. Paulo, 15:371-8, 1981.

pelo pessoal auxiliar de enfermagem, sob supervisão da enfermeira, aos clientes da área que não requeiram atenção imediata do médico ou da enfermeira.

- Desenvolver programas de educação em serviço para o pessoal de enfermagem sob responsabilidade da enfermeira.

- Prever 10 atendimentos de enfermagem à criança sadia, para controle do crescimento e desenvolvimento, na seguinte concentração:

3 atendimentos de enfermagem no 19 ano de vida exclusive;

7 atendimentos de enfermagem no período de um a 5 anos exclusive.

- Oferecer consultas de enfermagem e atendimentos de enfermagem para o controle do crescimento e desenvolvimento à criança menor de 5 anos que tenha recebido assistência médica inicial.

- Encaminhar para consulta médica, sempre que indicado, toda criança com problema de saúde.

Para Adami 1 (1976) as açōes mais significativas prestadas no período pré-patogênico e patogênico são a consulta de enfermagem e $o$ atendiniento de enfermagem institucional. Atualmente estas atividades encontram-se integradas nos programas de saúde materna e da criança, desenvolvidas em vários estabelecimentos de saúde de alguns Estados da Federação como parte técnica preventiva aplicada ao grupo materno-infantil. Entretanto, observa-se a tendência de se generalizar no país esta modalidade assistencial, com o propósito de ampliar os serviços de saúde oferecidos a esses grupos vulneráveis da população.

Yunes $^{3}$ (1975), na V Conferência Nacional de Saúde declara que "nos serviços de atendimento materno-infantil, a educação para a saúde é essencial e deve ser desenvolvida intensamente nas diferentes fases do programa. E necessário que as atividades educativas estejam perfeitamente integradas às demais. Todo o pessoal de saúde, no exercicio de sua profissão, deve realizar suas tarefas como educadores, seja na condição de médico, enfermeiro, dentista, nutricionista ou pessoal auxiliar. Entretanto, impōe-se o treinamento desse pessoal para as tarefas propostas, a fim de que possa realmente assumir as responsabilidades educativas que the compete". Esse autor recomenda ainda que "para a implantação do programa torna-se imperativa a formação e treinamento continuo de pessoal, quantitativa e qualitativamente adequado, para produzir as ações nele especificadas".

Levantada a opinião de vários autores sobre $o$ atendimento de enfermagem institucional, conhecida a opinião das funcionárias responsáveis pela execução da atividade e baseando-se que na grande maioria das vezes os atendimentos de enfermagem eram encaminhados para a consulta médica, levando com isso à duplicidade de atendimento médico-enfermagem, foi programado junto com a equipe técnica do CSEPM um treinamento e um roteiro específico para a adequada execução da atividade (Anexo).

\section{METODOLOGIA}

Foi realizado levantamento de atendimentos de enfermagem nos prontuários da clientela matriculada no Programa de Assistência à Criança do CSEPM no período de outubro de 1977 a outubro de 1978, com a finalidade de obter dados sobre:

- proporção de encaminhamentos à consulta médica

- proporção de casos em que se confirmou a necessidade da consulta médica.

Esse levantamento foi realizado em 113 prontuários antes de ser ministrado às visitadoras, encarregadas do atendimento, um treinamento específico para essa atividade. Posteriormente, esse levantamento foi repetido, em 312 casos, após o referido treinamento. 
MOURA, N. F. Estudo do atendimento de enfermagem institucional a crianças de 0 a 5 anos. Rev. Saúde públ., S. Paulo, 15:371-8, 1981.

Os resultados de cada fase do levantamento foram analisados estatisticamente pela técnica do $x^{2}$.

Concomitantemente e após o treinamento foi feito um estudo da duração média do atendimento, tendo sido cronometrados, para tanto, 168 atendimentos.

\section{RESULTADOS E DISCUSSAO}

A Tabela 1 mostra nos prontuários levantados antes (113) e depois (312) do treinamento, apenas nos casos em que as crianças foram encaminhadas ao médico, a confirmação ou não da necessidade desse encaminhamento.

\section{T A B E L A}

Crianças encaminhadas ao médico segundo a confirmação da necessidade de encaminhamento antes $e$ depois do treinamento.

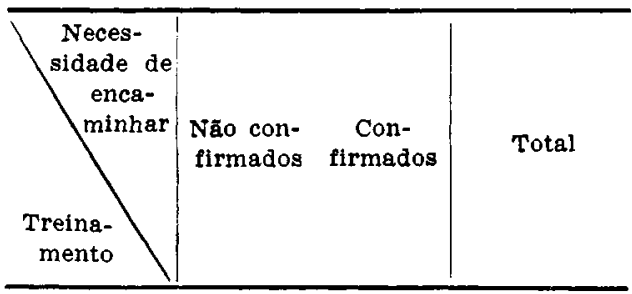

\begin{tabular}{lllr} 
Antes & 23 & 39 & 62 \\
Depois & 33 & 83 & 116 \\
\hline Total & 56 & 122 & 178 \\
\hline
\end{tabular}

Fonte: Dados do CSEPM.

Os atendimentos de enfermagem encaminhados ao médico representaram $41,9 \%$ do total levantado e, destes, $68,5 \%$ foram confirmados. Estes atendimentos confirmados antes do treinamento representaram $62,9 \%$, e após o treinamento $71,5 \%$.
Aplicando-se o teste de associação pelo Qui quadrado, chegou-se ao seguinte resultado:

$$
x^{2}{ }_{\mathrm{obs}}=1,03 \mathrm{e} x^{2}{ }_{\mathrm{crit}}(0,05)=3,84 .
$$
Como $\chi^{2}$ crítico ao nível de $5 \%$ é de 3,84 . conclui-se que não há associação (não houve alteração da proporção de necessidade não confirmada com o treinamento).

T A B E L A 2

Média de duração do atendimento de enfermagem.

\begin{tabular}{|c|c|}
\hline Minutos & No de atendimentos \\
\hline 5 & 0 \\
\hline 10 & 1 \\
\hline 15 & 27 \\
\hline 20 & 54 \\
\hline 25 & 42 \\
\hline 30 & 28 \\
\hline 35 & 9 \\
\hline 40 & 3 \\
\hline 45 & 3 \\
\hline 50 & 1 \\
\hline & 168 \\
\hline
\end{tabular}

Média de duração 23,8 min tos.

Conforme normas do Programa Materno-Infantil e Subprograma de Atendimento Médico e de Enfermagem - Área Infantil da Secretaria de Saúde do Estado de São Paulo, adaptado para o CSEPM, onde é prevista a duração de $15 \mathrm{~min}$ por atendimento de enfermagem, verifica-se uma defasagem de $8,8 \mathrm{~min}$ das normas padronizadas na programação e do tempo real de execução do atendimento, cronometrado durante a atividade. 
MOURA, N. F. Estudo do atendimento de enfermagem institucional a crianças de 0 a 5 anas. Rev. Saude públ., S. Paulo, 15:371-8, 1981.

T A B E L A 3

Opiniăo da clientela do Centro de Saúde Experimental da Escola Paulista de Medicina sobre o atendimento de enfermagem.

\begin{tabular}{|c|c|c|c|c|}
\hline \multirow[b]{2}{*}{ Respostas } & \multicolumn{2}{|c|}{ Fora da área } & \multicolumn{2}{|c|}{ Dentro da área } \\
\hline & No & $\%$ & No & $\%$ \\
\hline \multicolumn{5}{|l|}{ Escolaridade } \\
\hline analfabeta & 3 & 13,0 & 8 & 29,6 \\
\hline sabe ler e escrever & 3 & 13.0 & 1 & 3,7 \\
\hline primário incompleto & 11 & 47,8 & 10 & 37,0 \\
\hline acima do primário & 6 & 26,1 & 8 & 29,6 \\
\hline \multicolumn{5}{|l|}{ Tempo de freqüência no CSEPM } \\
\hline um ano ou menos & 19 & 82.6 & 4 & 14,8 \\
\hline mais de um ano & 4 & 17,4 & 23 & 85,2 \\
\hline \multicolumn{5}{|l|}{ Quem atende } \\
\hline médico & - & - & 2 & 7,4 \\
\hline visitadora & - & - & - & - \\
\hline ambos & 23 & 100,0 & 25 & 92,6 \\
\hline \multicolumn{5}{|l|}{$\begin{array}{l}\text { Sabe porque ds vezes so é } \\
\text { atendido por visitadora }\end{array}$} \\
\hline $\operatorname{sim}$ & 23 & 100,0 & 25 & 92,6 \\
\hline não & - & - & - & - \\
\hline não responde & - & - & 2 & 7,4 \\
\hline \multicolumn{5}{|l|}{ Em caso afirmativo, por que? } \\
\hline não tem médico & 1 & 4.3 & 2 & 8,0 \\
\hline médico ocupado & 2 & 8.7 & 2 & 3,0 \\
\hline criança näo tem problemas & 15 & 65,2 & 19 & 76.0 \\
\hline porque não é necessário & - & - & - & - \\
\hline todos os meses & - & - & - & - \\
\hline Outros & 5 & 21,8 & 2 & 8.0 \\
\hline \multicolumn{5}{|l|}{$\begin{array}{l}\text { Gosta que seja atendida pela } \\
\text { visitadora }\end{array}$} \\
\hline sim & 18 & 78,3 & 21 & 84,0 \\
\hline năo & 5 & 21,7 & 4 & 16,0 \\
\hline
\end{tabular}

Na Tabela 3 o número de entrevistadas fora da área perfaz o total de 23 , e dentro da área, 27.

A população da área é um pouco mais heterogênea com relação à escolaridade $\mathrm{e}$ frequienta o CSEPM há mais tempo.

Os demais resultados se equivalem, motivo pelo qual não foram realizados outros cruzamentos.
Com respeito à aceitação do atendimento de enfermagem, $81,2 \%$ de 48 entrevistadas (duas clientes não responderam) gostaram que a criança fosse atendida pela visitadora sanitária, quando não apresentasse problemas de saúde.

Como podemos notar na Tabela 4 algumas opiniões das visitadoras sanitárias após o treinamento modificaram-se. 


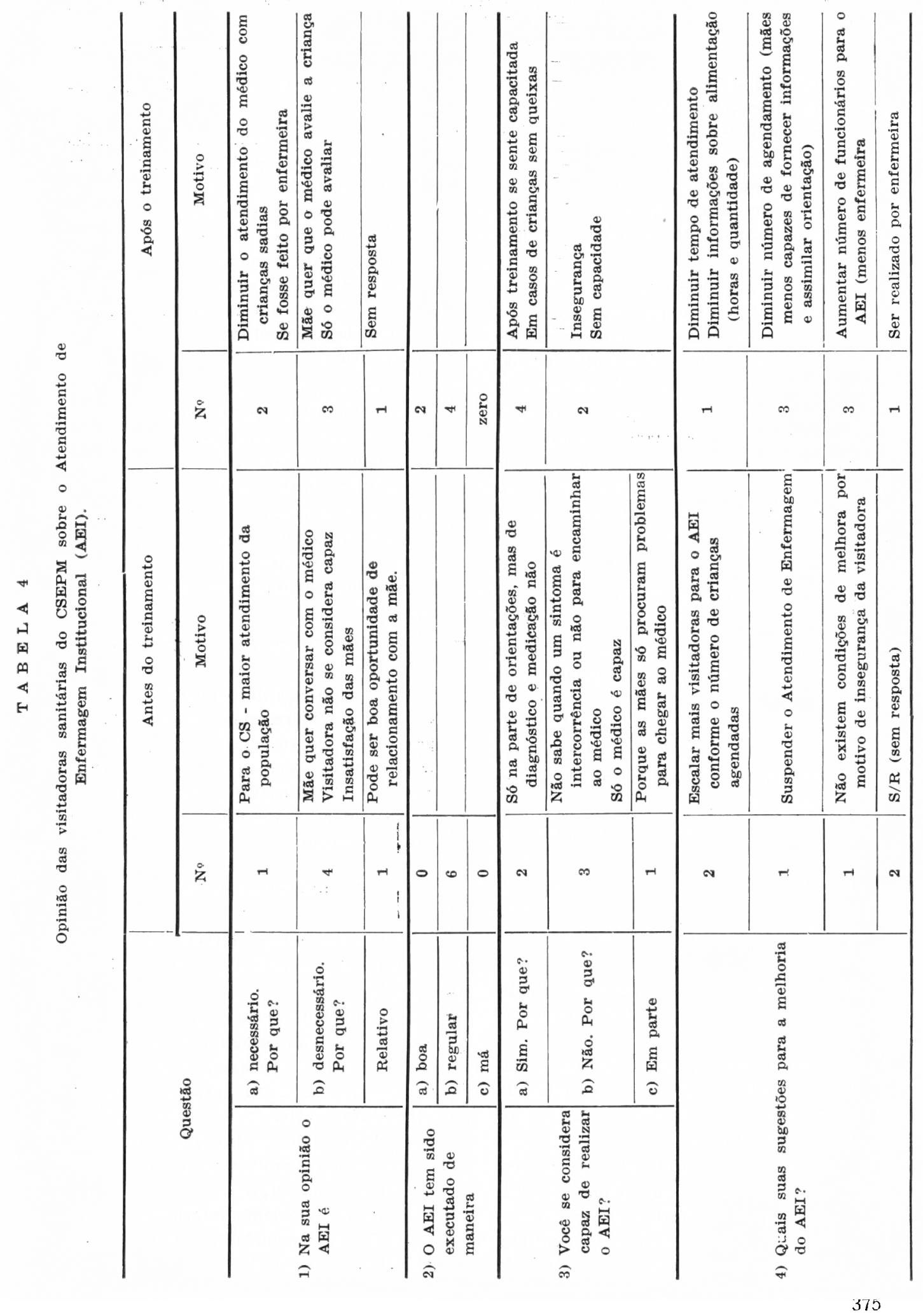


MOURA, N. F. Estudo do atendimento de enfermagem institucional a crianças de 0 a 5 anos.

Rev. Saúde públ., S. Paulo, 15:371-8, 1981

Segundo qualidade da execução do atendimento de enfermagem (boa), de zero antes do treinamento, foi para 2 após o treinamento.

Quanto à capacidade em realizar o atendimento de enfermagem, houve 2 respostas afirmativas antes do treinamento e 4 após o treinamento.

Quanto às sugestões para melhoria do atendimento, 4 visitadoras sanitárias demonstraram resistência à continuação do atendimento antes do treinamento: apenas uma opôs resistência após o treinamento.

Pode-se verificar, pois, que as opiniōes foram modificadas de maneira positiva a respeito do atendimento de enfermagem levando-nos a supor que esses resultados foram, em parte, devido à execução de um programa de treinamento.

\section{CONCLUSÕES}

De acordo com os resultados dos dados analisados, podemos tirar as seguintes conclusões:

4,1. Não assimilação, por parte das visitadoras sanitárias, dos objetivos e importância do atendimento de enfermagem por:

- mêdo de tomar decisões que poderiam implicar em riscos à saúde de criança,

- falta de treinamento no início do programa que demonstrasse o objetivo e os métodos de execução do atendimento de enfermagem,

- falta de incentivo, por parte da equipe para a execução do atendimento de enfermagem,

- falta de motivação por parte das visitadoras sanitárias para a execução do atendimento de enfermagem devido aos três primeiros itens.

4.2. As visitadoras sanitárias se sentem mais seguras e com maior conhecimento para realizarem o atendimento de enfermagem após o treinamento, o roteiro padronizado e a existência de retaguarda médica e de enfermagem.

4.3. Apesar das visitadoras sanitárias apresentarem opiniōes a respeito da não aceitação pelo público sobre o atendimento de enfermagem pudemos verificar, através das entrevistas realizadas, que a maioria das mães, ou seja $81,2 \%$, aceitam e gostam do atendimento apesar de nāo terem sido bem orientadas a respeito da atividade.

4.4. Antes e após o treinamento, os encaminhamentos para consulta médica continuaram a acontecer com a mesma freqüência, fato este que podemos explicar pelos seguintes motivos:

- falta de padronização do serviço e falta de segurança do elemento responsável para orientar casos de problemas que requeiram condutas puramente sintomáticas,

- falta de discernimento para definir intercorrências,

- falta de confiança da população no atendimento de enfermagem, motivado talvez pela falta de orientação adequada ou talvez pela insegurança sentida pelo elemento responsável pela atividade, muitas vezes transmitida no momento do atendimento.

\section{RECOMENDAÇOES}

As conclusões acima referidas nos sugerem pontos importantes a serem salientados tais como:

- Toda atividade a ser implantada em nivel de atenção primária à Saúde deve ser precedida de treinamento aos funcionários que a executarăo, e contínua supervisão, avaliação e reformulação.

- Deve haver uma padronização de condutas face aos problemas que rotineiramente possam surgir.

- Deve haver suficiente informação e divulgação à comunidade sobre a nova modalidade de atendimento. 
MOURA, N. F. Estudo do atendimento de enfermagem instit:cclonal a crianças de 0 a 5 anos. Rev. Saúde públ., S. Paulo, 15:371-8, 1981.

MOURA, N.F. [Institutional nursing attendance of children zero to five years of age]. Rev. Saúde públ.. S. Paulo, 15:371-8, 1981.

ABSTRACT: Operational evaluation of institutional nursing attendance given children 0-5 years of age was carried out from October, 1977 to October, 1978, in the Experimental Health Center of the "Escola Paulista de Medicina", S. Paulo (Brazil). It tells of problems that have arisen since the implanting of the Center and recounts clientes' opinions about this new vehicle of community health care.

UNITERMS: Health Centers. Child care. Nursing care. Evaluation.

\section{REFERENCIAS BIBLIOGRAFICAS}

1. ADAMI, N.P. A enfermagem de saúde pública na assistência progressiva ao paciente. Enferm. novas Dimens., 2(1):17-23 1976.

2. UNIVERSIDADE DE SRO PAULO. Faculdade de Saúde Pública. Disciplina de Enfermagem em Saúde Pública. Programa de assistenoia de saúde. São Paulo, s.d. [postila].
3. YUNES, J. Programa nacional de saúde materno-infantil. In: Conferência Na. cional de Saúde, 57, 1975. Anais. Brasilia, Ministério da Saúde, 1975. p. 45-134.

Recebido para publicagão em 05/09/1980

Aprovado para publicasão em 11/03/1981 
MOURA, N. F. Estudo do atendimento de enfermagem institucional a crianças de 0 a 5 anos. Rev. Saúde públ., S. Paulo, 15:371-8, 1981.

ANEXO

\section{ROTEIRO PARA EXECUÇAO DO ATENDIMENTO DE ENFERMAGEM INSTITUCIONAL}

- Sinais vitais (medir e registrar).

- Verificar peso e estatura comparando com o anterior. Caso não haja aumento desses valores pesquisar possiveis causas.

- Indagar da mãe o estado atual de saúde da criança:

1 - Alimentação

2 - Imunização

3 - Eliminação

4 - Hábitos de Saúde

5 - Crescimento e Desenvolvimento

- Ouvir e registrar possiveis queixas sobre saúde da criança.

\section{1 - Alimentação}

- Verificar a alimentação, sem induzir nenhum alimento, recordando o que a criança comeu nas últimas 24 horas.

- Diluição do leite (quantidade de água, n? de medidas do leite, açúcar e/ou mamadeiras).

- Quantidade de leite/dia

- Refeição de sal

- Fruta

- Verificar, apenas perguntando, se a criança toma alguma "vitamina" (vit. A e D e sulfato ferroso). No pensamento popular o ferro também é vitamina.

- quantidade

- época de início.

- Orientar alimentação seguindo esquema simplificado.

- Orientar a suplementação de vitamina e ferro seguindo a rotina.

- Registrar na pasta o encaminhamento de suplemento alimentar (leite em pó).

\section{2 - Vacinação}

- Verificar carteira de vacinação detectando as doses a serem aplicadas.
- Orientação à paciente sobre significado, importância, cuidados e reaçōes das vacinas a serem aplicadas.

- Encaminhamento à sala de vacinação.

Obs.: Orientar a paciente para que traga a carteira de vacinação sempre que vier à Unidade.

3 - Eliminações

- Fezes

$$
\begin{aligned}
& n \% \text { de vezes } \\
& - \text { consistência } \\
& - \text { odor } \\
& - \text { cor }
\end{aligned}
$$

- Urina

$$
\begin{aligned}
& \text { - odor } \\
& \text { - cor } \\
& \text { - quantidade }
\end{aligned}
$$

- Regurgitação

$$
\text { - existência e características }
$$

Obs.: Orientar caso haja qualquer alteração.

4 - Hábitos de Saúde

- Banho de sol

- Sono

- Higiene

- Passeios

- Brinquedos

5 - Crescimento de Desenvolvimento

- Verificar desenvolvimento na escala de Desenvolvimento Motor de Shirley e crescimento através da Tabela de Gomes.

- Orientar e registrar erros de conduta.

- Requisitar a presença da enfermeira para dissipar dúvidas para posterior conduta.

Obs.: Somente a enfermeira encaminhará ao médico, se necessário.

Registrar em vermelho o atendimento de enfermagem. 